【电子与信息科学 / Electronics and Information Science】

\title{
量子保密通信与量子计算
}

\author{
郭邦红 ${ }^{1,2}$ ，胡 敏 ${ }^{2}$ ，毛 点 ${ }^{1}$ ，陈国良 ${ }^{1,3}$
}

1）深圳大学计算机与软件学院，广东深圳 $518060 ； 2$ ) 华南师范大学信息光电子科技学院，广东省微纳光子功能材料与

器件重点实验室, 广东广州 510631；3) 南京邮电大学高性能计算与大数据处理研究所，江苏南京 210003

摘 要: 量子保密通信与量子计算作为战略性前沿技术, 与国家安全和前瞻性技术战略关系密切, 未 来量子计算机的应用必将极大推动量子网络规模化应用. 本文综述分析量子保密通信与量子计算的研究进 展，重点介绍高性能量子密钥分发 (quantum key distribution, QKD) 终端、量子网络和量子计算领域在量子 芯片器件和量子位操控等方面的基础科学问题，以及量子与经典通信融合等实用量子关键技术.

关键词：量子调控; 量子保密通信; 量子网络; 高性能量子密钥分发; 量子计算机; 量子算法

中图分类号：043; TN918 文献标志码：A doi：10.3724/SP. J. 1249.2020.06551

\section{Recent progress in quantum cryptography and quantum computation}

\section{GUO Banghong ${ }^{1,2}$, HU Min ${ }^{2}$, MAO Rui ${ }^{1}$, and CHEN Guoliang ${ }^{1,3}$}

1) College of Computer Science and Software Engineering, Shenzhen University, Shenzhen 518060, Guangdong Province, P. R. China

2) School of Information and Optoelectronic Science and Engineering, Guangdong Provincial Key Laboratory of Nanophotonic

Functional Materials and Devices, South China Normal University, Guangzhou 510631, Guangdong Province, P. R. China

3 ) Research Institute of High Performance Computing and Big Data Processing, Nanjing University of Posts and

Telecommunication, Nanjing 210003, Jiangsu Province, P. R. China

\begin{abstract}
As a strategic frontier technology, quantum cryptography and quantum computation are closely related to national security and forward-looking technology strategy, and the application of quantum computation in the future will definitely promote the large-scale application of quantum networks. We briefly review the research progress of quantum secure communication and quantum computing and focus on the introduction of the high-performance quantum key distribution ( QKD) terminal, quantum network, basic scientific problems of quantum computing in quantum chip devices and qubits manipulation and some practical important quantum technologies such as the integration of quantum and classical communication.
\end{abstract}

Key words: quantum control; quantum secure communication; quantum network; high-performance quantum key distribution; quantum computer; quantum algorithm

量子保密通信与量子计算是当前国际量子物理 和信息科学的研究热点. 世界各国都在战略部署量
子保密通信与量子计算基础研究, 中国也已将新一 代量子通信与网络、量子科学、脑科学和人工智能

Received: 2020-03-31; Accepted: 2020-06-24

Foundation: National Natural Science Foundation of China (61572203) ; Key-Area Research and Development Program of Guangdong Province (2018B030325002)

Corresponding author: Postdoctoral researcher GUO banghong. E-mail: guobangh@163.com

Citation: GUO Banghong, HU Min, MAO Rui, et al. Recent progress in quantum cryptography and quantum computation [ J]. Journal of Shenzhen University Science and Engineering, 2020, 37(6) : 551-558. (in Chinese) 
等作为前沿科学领域布局. 自 BENNETT 和 BRASSARD 提出 BB84 协议以来 ${ }^{[1]}$ ，基于量子密钥分发 (quantum key distribution, QKD) 的量子保密通信技 术得到了迅速的发展. 量子保密通信与量子计算作 为战略性前沿技术, 关系国家安全, 正在推动全球 经济发展和社会进步, 对军事、经济、金融和社会 等各领域安全带来深远影响.

\section{1 量子保密通信与量子计算关键技术}

当前，世界正在兴起新一轮 “量子革命”，量 子信息与量子计算技术正处在加速突破关键技术的 新阶段, 特别是高性能 QKD 终端正逐步在系统集 成、工程化和网络化等关键技术方面取得突破，量 子保密通信与经典通信网络融合、量子计算机多位 (bit) 操作和运算已显现出量子信息和量子网络的产 业化应用前景.

\section{1 高性能 QKD 终端技术}

1989 年，BENNETT C H 和 BRASSARD G 基于 BB84 协议实现了世界首个 QKD 原理实验，遗憾的 是速率只有 $10 \mathrm{bit} / \mathrm{s}$, 传输距离仅 $32 \mathrm{~cm}$. 经过 30 多年的发展, QKD 在传输距离、速率和成码率等关 键技术方面屡获突破，基于光纤点对点无中继 QKD 的安全传输距离已超 $250 \mathrm{~km}^{[2-3]}$, 而基于纠缠光子 对 QKD 的传输距离也已达到 $300 \mathrm{~km}^{[4-5]}$. 这类 $\mathrm{QKD}$ 解决了长距离传输的技术难题, 但工程化精密 控制弱脉冲和弱信号探测技术难度大．测量设备无 关 QKD (measurement device independent QKD, MDI QKD) 的传输距离超过 $400 \mathrm{~km}$, 具有兼顾安全性和 实用性的技术优势 ${ }^{[6]} .2020$ 年，王向斌团队报道了 双场量子密钥分发 (twin-field QKD, TF-QKD) 实验, 其安全传输距离达 $509 \mathrm{~km}^{[7]}$.

量子安全直接通信 (quantum secure direct communication, QSDC) 是一种不同于 QKD 的量子通信 形式, 是量子保密通信的一个重要分支. QSDC 采 用低密度奇偶校验码直接传送秘密信息，可在高噪 声和高损耗的实际环境中工作，实验中重复频率为 $1 \mathrm{MHz}$ ，安全通信速率达 $50 \mathrm{bit} / \mathrm{s}$. 尽管可有效发送 文本、较小容量的图像和声音文件, 但其传输距离 仅为 $1.5 \mathrm{~km}$, 且采用了超导探测器 ${ }^{[8]}$.

QKD 系统的极弱量子信号易受自发辐射噪声和 探测器暗计数、光纤损耗及 $Q K D$ 编解码器件等引 人噪声的影响. 尽管目前通过采用相位调制补偿、 法拉第旋转镜 (Faraday rotator mirror, FM) 等方法后
有所改善，但是牺牲了部分内禀光子 ${ }^{[9]}$. 相位偏振 联合调制 QKD 系统内禀光子利用率由 0.5 提到 2.0 , 提高了 3 倍 ${ }^{[10]}$.

因此, 开发高性能 QKD 系统, 解决能够高码 率且稳定运行等技术难题是实现 QKD 网络应用的 重要任务, 而获得安全传输距离为数百 $\mathrm{km}$ 量级密 钥的 QKD 终端设备是网络接人的基础条件，集成 化、小型化 QKD 更是未来产业应用的发展趋势.

\section{2 量子计算与量子计算机}

1965 年，MOORE 提出当价格不变时，集成电 路 (integrated circuit, IC) 上可容纳的元器件的数目, 每隔大约 $18 \sim 24$ 个月便会增加 1 倍，性能也将提 升 1 倍, 即著名的摩尔定律. 理论研究表明, 由于 经典计算是不可逆的，因此，每删除 1 bit 信息至 少要耗散 $k T \ln 2$ 的能量 $(k$ 为玻尔兹曼常数, $T$ 为该 bit 所在环境的开尔文温度)，从而导致 IC 存在理 论极限问题. 基于量子力学原理的量子位 (qubit) 逻 辑门计算相比传统经典位计算具有极大的优势. 量 子力学的么正演化结构逻辑门, 即量子计算么正演 化，具有可逆操作和可逆计算的特点，有望解决当 前 IC 能量耗散导致的极限问题.

量子计算的主要目的之一是在特定领域实现经 典计算无法实现的功能. 1982 年，FEYNMAN R 提 出量子计算概念，他认为微观世界的本质是量子 的, 可建造一个根据量子力学规律运行的计算机, 用于模拟量子世界的部分行为. 量子计算需突破物 理和化学基础新材料等关键技术，解决量子芯片、 量子位操控、量子计算机物理实现及软件体系等难题.

\subsection{1 量子芯片器件}

量子计算与量子通信是《“十三五” 国家科技 创新规划》的重要战略方向, 规划强调要推进量子 通信城域和城际网络等重大应用. QKD 集成化、小 型化和抗噪声是量子信息技术产业应用和城域、城 际网络规模应用亟需解决的关键技术问题.

当前 $Q K D$ 系统采用分立器件, 体积较大, 光 源弱脉冲的精密控制和探测实验技术工程化难度 大. 以集成光学为技术方案的量子器件集成和量子 信息处理成为研究热点. LIU 等 ${ }^{[11]}$ 提出一种波导集 成量子器件, 可实现保真度为 0.93 的轨道角动量 转换，通过控制人射光的相位高效灵活地调制轨道 角动量 (图 1). MARTIN 等 ${ }^{[12]}$ 提出 $766 \mathrm{~nm}$ 光源和 集成量子耦合光路的量子芯片（图 2)，利用光通 信集成技术实现量子中继芯片.

2017 年, SPRING 等 ${ }^{[13]}$ 在二氧化硅芯片上集成 


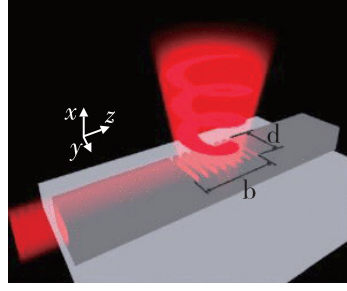

（a 集成波导浴旋光束发生器

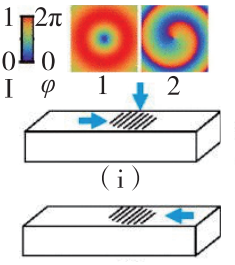

(iii)
图 1 集成波导涡旋光束发生器的示意图与 波导中的全息光栅原理 ${ }^{[11]}$

Fig. 1 Schematic illustration of the proposed vortex beam generator on the integrated waveguide and the principle of the holographic grating on waveguide ${ }^{[11]}$

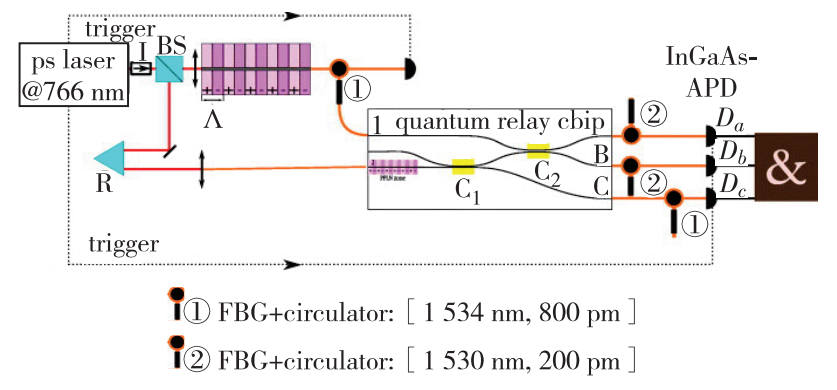

图 2 表面 PPLN 波导源量子中继芯片实验示意图 ${ }^{[12]}$

Fig. 2 Schematic experiment of surface PPLN waveguide source quantum relay chip ${ }^{[12]}$

了 5 个宣布式单光子源阵列, 其二阶自相关函数 $g^{2}(0)=0.03 \pm 0.01$, 重复频率约为 $200 \mathrm{kHz}$. 这表 明可将多个宣布式单光子源集成在单个芯片上, 以 提高重复频率. BELHASSEN 等 ${ }^{[14]}$ 基于 III-V 族材 料, 将宣布式单光子源和分束器集成在单一芯片 上, 并进行 Hanbury-Brown and Twiss ( HBT) 实验, 获得二阶自相关函数 $g^{2}(0)=0.10 \pm 0.02$, 但是重 复频率只有 $200 \mathrm{~Hz} .2016$ 年, WANG 等 ${ }^{[15]}$ 在集成 光子芯片系统中实现了路径-偏振转换, 6 个量子态 的平均保真度为 $98.82 \pm 0.73 \%$. 2017 年, SIBSON 等 ${ }^{[16]}$ 制作了基于 $\mathrm{InP}$ 的 QKD 发射端和基于氮氧化 硅的 QKD 接收端, 经过 $20 \mathrm{~km}$ 光纤传输后实现了 $1.05 \%$ 的低量子误码率. 2019 年, 潘建伟等 ${ }^{[17]}$ 提 出超低损耗光路的三维集成 Boson 采样方案(图 3), 在 60 模干涉仪中进行了 20 个单光子的实验.

在无源器件方面, 可在硅基材料上设计集成微 米尺度的耦合器、分束器、相位调制器、微环滤波 器、光延时线和阵列波导光栅等分立元器件. 郭邦 红等在互补金属氧化物半导体 (complementary metal oxide semiconductor, CMOS) 的集成光学平台上研究

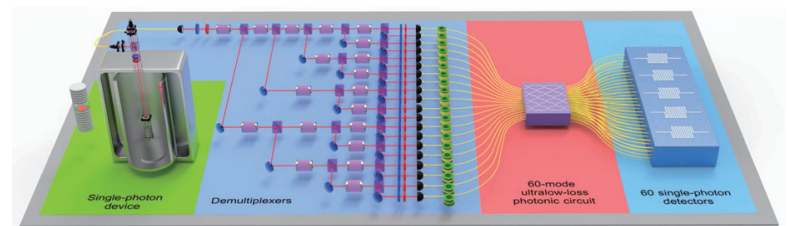

图 3 三维集成 Boson 采样芯片 ${ }^{[17]}$

Fig. 3 Three-dimensional integrated Boson sampling chip

了多种纳米波导, 如基于纯电解质波导的超宽带波 片 $^{[18]}$ 和能够实现单向轨道角动量转换的集成波 片 $^{[19]}$. YANG 等 ${ }^{[20]}$ 研究了硅基多模波导集成的马 赫-曾德尔 (Mach-Zehnde, M-Z) 干涉仪, 其消光比 大于 $20 \mathrm{~dB}, 30 \mathrm{~nm}$ 波长范围内波长相对于温度的 稳定性为 $\pm 10 \mathrm{pm} / \mathrm{K}$.

在利用超导量子芯片实现量子计算方面, 因为 加工技术成熟, 易于扩展, 且集成度高, 具有较好 的应用前景, 目前主要是基于约瑟夫结实现超导量 子位. 加拿大 D-wave 公司 2000 量子位的量子退火 机、美国 Google 公司 72 量子位的量子计算机以及 IBM 公司的量子计算机是该方案的典型代表.

采用经典(非可逆)计算方式的芯片在计算时一 定伴随着能量的耗散, 随着芯片集成度的急剧增 加, 热效应将更加显著, 最终导致芯片损坏, 于是 IC 将达到极限尺寸. 另外, 如果尺度继续减小就到 了量子力学的适用范围, 量子芯片器件必将成为未 来量子科学应用领域的关键技术.

\subsection{2 量子位操控}

已有多种方法可实现量子位操控, 如核磁共振 (nuclear magnetic resonance, NMR)、光量子、贝禁离 子和超导等. 经过 50 年的发展, 核磁共振对耦合 二能级量子系统的动力学控制达到了新的水平. 目 前, 基于核磁共振技术的量子控制和计算可精确地 控制达 12 个量子位的系统 ${ }^{[21]}$, 如龙桂鲁教授团队 开发的基于核磁共振体系的量子计算云平台 ${ }^{[22]}$.

光量子计算方案基于光子自由度（偏振、空间 和轨道角动量等), 采用线性光学元件作为量子门, 通过单光子探测器实现量子测量. WANG 等 $^{[23]}$ 利 用 6 个光子的 3 个自由度实现了 18 个量子位的操 控. TANG 等 ${ }^{[24]}$ 制作了 $49 \times 49$ 个节点的光子芯片, 并在该芯片上实现了两维的量子行走.

1995 年 CIRAC 等 ${ }^{[25]}$ 提出, 可以使用激光将离 子冷却并囚禁于真空中, 则该离子就可作为量子位 进行基本的量子逻辑门操作. 因为同时满足量子计 
算 5 个基本准则和量子网络 2 个准则 ${ }^{[26]}$, 囚禁离子 被认为是最有希望实现大规模量子计算和量子网络 的物理系统. 美国 IonQ 公司和马里兰大学团队 ${ }^{[27]}$ 基于 13 个 $^{171} \mathrm{Yb}^{+}$构成的囚禁离子系统，实现了 11 个量子位全连接的可编程量子计算机, 宣称采用相 同装置，成功加载了超过 150 个离子，并在高达 79 个量子位中进行选择性的单量子位旋转. 清华大学 金奇负研究组将囚禁离子系统的退相干时间延长到 了 $10 \mathrm{~min}^{[28]}$, 并实现了任意量子位之间的全局纠 缠门 ${ }^{[29]}$.

在超导量子位操控方面，加拿大 D-wave 公司 2011 年发布了具有 128 个量子位的量子计算机, 2017 年发布的 D-wave 2000Q 系统具有 2000 个量子 位 ${ }^{[30]}$ 。但 D-wave 公司的量子计算机是基于量子隧 穿效应实现的量子退火 (quantum annealing)，与通 用量子计算机差异较大. Google 和 IBM 公司在超导 量子位操控方面目前处于领先地位，二者已经分别 可以控制 $72 \mathrm{bit}$ 和 $50 \mathrm{bit}^{[31]}$. 在中国, 本源量子计 算科技有限责任公司发布了超导量子计算云平 台 ${ }^{[32]}$, 浙江大学等多家单位合作实现了 20 个量子 位操控 ${ }^{[33]}$.

由于量子叠加原理, $n$ 量子位可同时处于 $2^{n}$ 种 状态 (一个量子位的任意状态可用图 4 中布洛赫球 上的点来表示)，当量子计算结束时，通过量子测 量, 这 $2^{n}$ 种状态将均塌到其中一种确定的状态，如 此完成并行计算

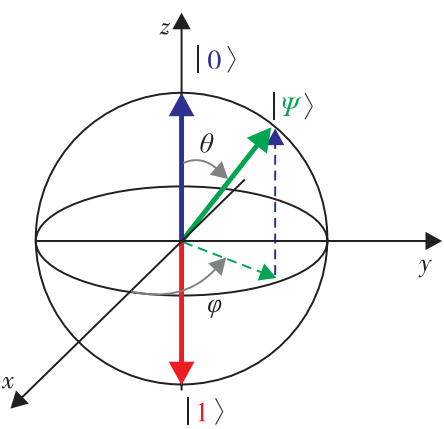

图 4 布洛赫球中量子比特

Fig. 4 Qubits in a Bloch sphere

\subsection{3 量子计算与量子计算机}

1996 年，SHOR ${ }^{[34]}$ 提出一个能在多项式时间内 求解给定整数质因子的量子算法. GROVER ${ }^{[35]}$ 提出 GROVER 量子搜索算法. 表 1 是几种典型的量子算 法的实验实现 ${ }^{[36]}$.

量子纠缠叠加原理使量子位比经典位能处理更 多信息，这就是量子计算机相对于经典计算机的优
势. 量子计算机的 1 次操作同时完成了对 $2^{n}$ 个数据 的操作，相当于经典计算机完成了对 $2^{n}$ 个数据的并 行处理. 基于量子力学原理的量子计算机是下一代 计算革命的关键所在.

表 1 几种量子算法解决 “最大” 问题的实验实现 ${ }^{[36]}$

Table 1 Experimental implementation of several quantum algorithms to solve the "biggest" problem ${ }^{[36]}$

\begin{tabular}{|c|c|c|}
\hline 算法 & 实验技术 & 解决的问题 \\
\hline SHOR 算法 & 块状光学器件 ${ }^{[37]}$ & $\begin{array}{l}\text { 数字 } 21 \text { 的 } \\
\text { 质因子分解 }\end{array}$ \\
\hline GROVER 算法 & 核磁共振 $\mathrm{NMR}^{[38]}$ & $\begin{array}{c}\text { 非结构化搜索, } \\
\text { 数据库记录数 } \\
\quad N=8\end{array}$ \\
\hline 量子退火算法 & D-wave $2 X^{[39]}$ & $\begin{array}{c}1097 \text { 个顶点的 } \\
\text { “Chimera” 图 } \\
\text { Ising 模型 }\end{array}$ \\
\hline $\begin{array}{l}\text { HHL( Harrow, } \\
\text { Hassidim and } \\
\text { Lloyd) 算法 }\end{array}$ & $\begin{array}{c}\text { 块状光学 } \\
\text { 器件 } \\
\mathrm{NMR}^{[42-41]},\end{array}$ & $2 \times 2$ 线性方程组 \\
\hline
\end{tabular}

LARSEN 等 ${ }^{[43]}$ 通过实验产生了 $3 \times 10^{4}$ 个纠缠 模式的二维簇态 (图 5), 可用于连续变量的量子计 算. IBM 和 Google 公司在量子计算机研究领域处于 领先地位. 2017 年 4 月, Google 公司开发出 9 个量 子位的量子计算芯片; 5 月 IBM 公司推出 17 个量 子位的量子计算芯片; 10 月 17 日 Intel 公司发布了 17 个量子位的超导测试芯片；同日，IBM 公司宣布 量子计算已经突破了 49 个量子位的障碍.

2017 年，潘建伟研究团队宣布通过将时间编码 的单光子序列输人电控可编程多模网络后, 实现了 可用于多光子 “玻色取样” 任务的光量子计算模拟 机 ${ }^{[44]}$. 2019 年，该团队开发了高效、纯、不可分 辨的单光子固态光源和超低损耗的三维集成光路. 他们在 60 模干涉仪中注人 20 个纯单光子 (图 6), 在输出段探测到了 14 个光子, 并且采样的希尔伯 特空间高达 $3.7 \times 10^{14}$, 该实验为通过玻色子取样 实现 NISQ 体系提供了一条新的途径 ${ }^{[17]}$.

2018 年，PRESKILL ${ }^{[31]}$ 提出有噪声中规模量子 ( noisy intermediate-scale quantum, NISQ) 概念, 认为 拥有 $50 \sim 100$ 量子位和高保真量子门 ( quantum gate) 的计算机, 便可称为 NISQ 计算机. NISQ 器件 将是探索多体量子物理的有用工具，虽然也可能有 其他的应用，但 100 量子位的计算机不会马上改变 世界。 


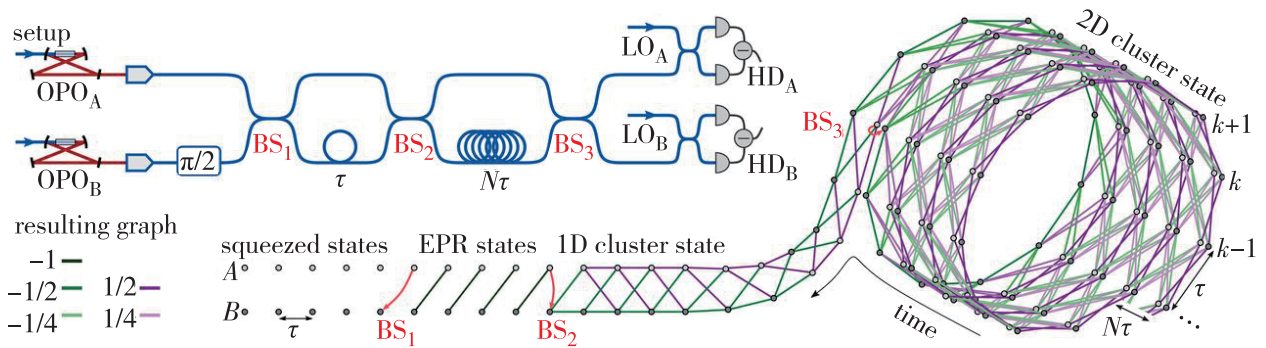

图 5 超规模纠缠二维集群纠缠点量子计算 ${ }^{[43]}$

Fig. 5 Quantum calculation of entangled points in a two-dimensional entangled cluster of hyperscale ${ }^{[43]}$

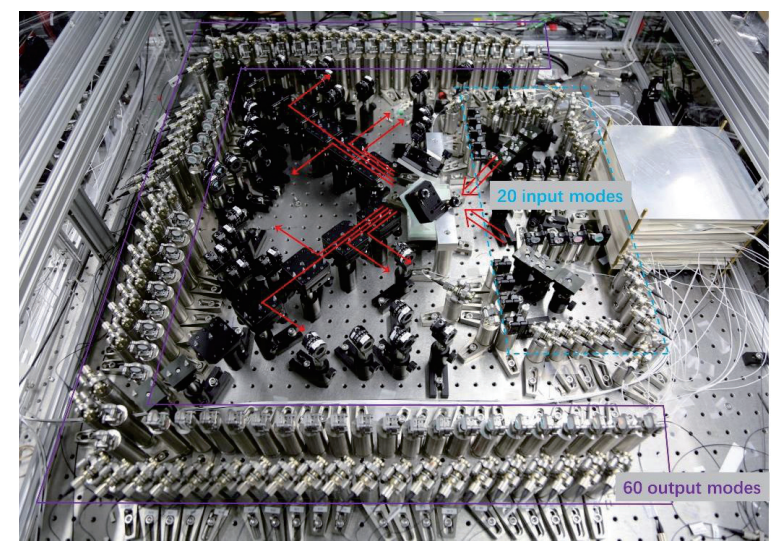

图 660 模干涉仪中进行了 20 个单光子实验 ${ }^{[17]}$

Fig. 620 single-photon experiments performed in a 60-mode interferometer ${ }^{[17]}$

加拿大 D-wave One 系统利用了量子隧穿效应 实现了量子退火，可将组合优化问题求解的非确定 性多项式 (non-deterministic polynomial, NP) 难题在 多项式时间解决，预计可以减少密码攻击所需搜索 空间的量级.

2019 年 8 月，浙江大学等单位组成的团队开发 出了具有 20 个超导量子位的量子芯片, 并用于产 生 18 个量子位的 GHZ( Greenberger-Horne-Zeilinger) 态和 20 个量子位的薛定谔猫态 ${ }^{[33]}$. 同年 10 月, ARUTE 等 ${ }^{[45]}$ 报道开发出了 “Sycamore” 量子芯片 (图 7), 包含 54 个 transmon 量子位的 2 维阵列, 其中每个量子位能够可调地被堣合到矩形晶格中 4 个最近相邻的量子位, 因此, 用于计算的希尔伯特 空间维度为 $2^{53} \approx 9 \times 10^{15}$. 实际上有 1 个量子位无 法正常工作，因此能够用于计算的只有 53 个量子 位和 86 个耦合器. ARUTE 等声称经典计算机需要 约 10000 年才能完成的量子电路取样任务采用该量 子处理器仅需要 $200 \mathrm{~s}$. 但是该工作后续引发了较 多争议, 最终并未获得学术界的认可. 11 月, 李传 峰等 ${ }^{[46]}$ 将机器学习应用于量子力学基础研究, 首

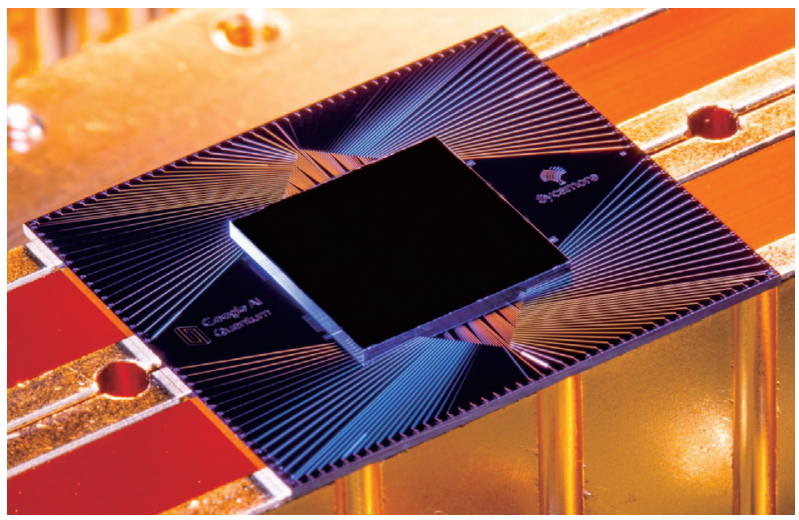

图 7 Sycamore 量子芯片 ${ }^{[45]}$

Fig. 7 Sycamore quantum chip ${ }^{[45]}$

次实现了基于机器学习算法的多重非经典关联.

过去 10 年中，量子计算已经从基础研究逐步 迈人工程化和商用化进程. 显然，量子计算仍将继 续取得进展，但是很难预测其发展路径和速度. 也 许量子计算会缓慢而渐进地发展，又或者会突然出 现意想不到的创新进而取得快速进步.

\section{2 量子保密通信与经典通信网络融合}

QKD 系统的网络应用是量子保密通信的产业发 展目标. 1994 年，TOWNSEND 等 ${ }^{[47]}$ 提出多用户量 子密钥分发网络, 采用光功率分配器构建了基于无 源光网络 (passive optical network, PON) 的量子网 络. 2003 年，BRASSARD 等 ${ }^{[48]}$ 提出采用波分复用 (wavelength division multiplexing，WDM) 技术的基于 光纤的量子密钥分发网络. 近年来还报道了其他几 类 QKD 的量子网络应用，如美国国防部联合多家 研究机构提出的美国国防高级研究计划局 (Defense Advanced Research Projects Agency, DARPA) 量子网 络 ${ }^{[49]}$, 这是世界首建的军事应用类量子网络. 当 前对量子网络的应用仍襄贬不一，有研究构建且验 
证了基于 QKD 接人的量子通信网络的可行性, 推 动了针对量子通信网络的研究和发展, 为量子通信 网络提供了理论依据和产业应用的参考，如欧盟资 助的 Secure communication based on quantum cryptography (SECOQC) 网 $^{[50]}$ ，刘颂豪团队基于教育骨干 网建设的天河区-番禺区量子网，郭光灿研究小组 的北京网通的商用光纤骨干网络和芜湖量子政务 网 $^{[51-52]}$, 潘建伟团队的量子通信网络 ${ }^{[33-54]}$, 以及日 本和欧洲等 9 个单位在日本建设的 Tokyo QKD 量子 网络 ${ }^{[55]}$. 2017 年中国科学院宣布开通京沪量子试 验干线网络. 次年, 沪杭试验干线开通测试. 2019 年，刘颂豪团队联合启动广佛肇 QKD 示范量子网 络（图 8). 该量子网络由广州城域量子网络、佛 山节点及肇庆城域量子网络构成，成为中国华南地 区首批量子安全通信网络, 理论规划光纤星地一体 6 个闭环网，成码速率不低于 $16 \mathrm{kbit} / \mathrm{s}$, 并将在政 务、金融或其他行业进行示范应用，是粤港澳大湾 区量子通信首条示范干线，其成果对建设粤港澳大 湾区量子通信环网具有重要意义 ${ }^{[56]}$.

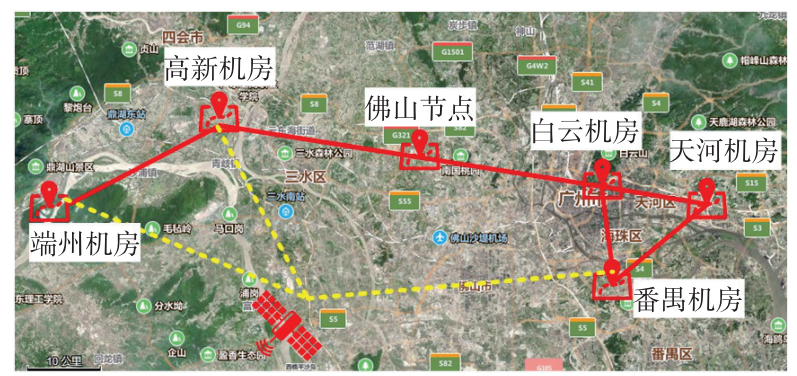

图 8 广佛肇量子干线网络

Fig. 8 Guang-fo-zhao quantum backbone network

当前量子网络的光接人网络方案都是单独或同 时采取基于可信节点的中继方案和基于光开关的透 明光链路. 前者可任意扩展密钥分发的距离，但必 须保证所有节点的物理安全; 后者可在非可信的网 络中实现多用户之间的密钥分发.

$\mathrm{SU}$ 等 ${ }^{[57]}$ 研究表明, 量子信号传输距离和密钥 生成率都受到光路损耗和噪声干扰的限制. 量子经 典融合网络须解决量子信号与经典信号如何共存共 纤传输技术问题，亟需发展网络接人的基础理论. 另外，机载 QKD 终端技术、空天一体化通信是未 来天地一体化通信的产业应用和国际量子网络的技 术挑战.

\section{结 语}

基于量子力学原理的量子计算机是下一代计算 革命的关键，未来量子计算机的应用必将极大地推 动量子网络的规模化应用. 当前量子计算机需要突 破更科学的物理实现机制和更高的量子位控制等关 键技术. 尽管世界各国的科学家们已经在量子计 算、量子调控和量子网络应用等领域取得了极大成 就，但在实现通用量子计算机、有效的量子算法、 长距离 QKD 稳定传输、多自由度的调制、量子网 络干线、机载量子纠缠密钥分发、天地一体化通信 和洲际通信等方面依然面临极大的挑战.

基金项目：国家自然科学基金资助项目（61572203）; 广东省重点 领域研发计划资助项目 (2018B030325002)

作者简介：郭邦红 (1975一), 深圳大学博士后研究人员. 研究方 向：量子通信与量子计算. E-mail: guobangh@ 163.com

引

文: 郭邦红, 胡 敏, 毛 㤐, 等. 量子保密通信与量子计 算 $[\mathrm{J}]$. 深圳大学学报理工版, $2020,37(6): 551-558$.

\section{参考文献 / References:}

[ 1 ] BENNETT C H, BRASSARD G. Quantum cryprography: public key distribution and coin tossing $[\mathrm{C}] / /$ Proceedings of IEEE International Conference on Computers, Systems and Signal Processing. Bangalore, India: Institute of Electrical and Electronics Engineers, 1984: 175-179.

[ 2 ] STUCKI D, WALENTA N, VANNEL F, et al. High rate, long-distance quantum key distribution over $250 \mathrm{~km}$ of ultra low loss fibres [J]. New Journal of Physics, 2009, 11(7) : 075003

[ 3 ] WANG Shuang, CHEN Wei, GUO Junfu, et al. $2 \mathrm{GHz}$ clock quantum key distribution over $260 \mathrm{~km}$ of standard telecom fiber [J]. Optics Letters, 2012, 37(6) : 1008 1010.

[ 4 ] INAGAKI $\mathrm{T}$, MATSUDA $\mathrm{N}$, TADANAGA $\mathrm{O}$, et al. Entanglement distribution over $300 \mathrm{~km}$ of fiber [ J ] Optics Express, 2013, 21(20) : 23241-23249.

[ 5 ] INAGAKI T, MATSUDA N, TADANAGA 0 , et al. Long distance distribution of entangled photon pair over $300 \mathrm{~km}$ of fiber [ C ]// CLEO: 2013. Washington D C: OSA, 2013: QTu2C. 2.

[ 6 ] YIN Hualei, CHEN Tengyun, YU Zongwen, et al. Measurement-device-independent quantum key distribution over a $404 \mathrm{~km}$ optical fiber [ J ]. Physical Review Letters, 2016, 117(19) : 190501

[ 7 ] CHEN Jiupeng, ZHANG Chi, LIU Yang, et al. Sending- 
or-not-sending with independent lasers: secure twin-field quantum key distribution over $509 \mathrm{~km}[\mathrm{~J}]$. Physical Review Letters, 2020, 124(7): 070501.

[ 8 ] QI Ruoyang, SUN Zhen, LIN Zaisheng, et al. Implementation and security analysis of practical quantum secure direct communication $[\mathrm{J}]$. Light: Science \& Applications, 2019, 8(1): 228.

[9] 苗二龙, 莫小范, 桂有珍, 等. 相位调制自由空间量 子密钥分配 $[\mathrm{J}]$. 物理学报, $2004,53(7)$ ：21232126.

MIAO Erlong, MO Xiaofan, GUI Youzhen, et al. Phasemodulated free space quantum key distribution $[\mathrm{J}]$. Acta Physica Sinica, 2004, 53(7) : 2123-2126. (in Chinese)

[10] 郭邦红, 张盼盼, 胡 敏. 位相偏波多自由度变調 QKDネットワークシステム及びその方法. 特願 2019-502561[P]. 2017-12-20.

GUO Banghong, ZHANG Panpan, HU Min. Phase and polarization multi-degree-of-freedom modulated QKD network system and method. Japanese patent: 2019502561. [P]. 2017-12-20. (in Japanese)

[11] LIU Aiping, ZOU Changling, REN Xifeng, et al. On-chip generation and control of the vortex beam $[\mathrm{J}]$. Applied Physics Letters, 2016, 108(18): 181103.

[12] MARTIN A, ALIBART O, DE MiCHELI M P, et al. A quantum relay chip based on telecommunication integrated optics technology [ J]. New Journal of Physics, 2012, 14 (2) : 025002.

[13 ] SPRING J B, MENNEA P L, METCALF B J, et al. Chipbased array of near-identical, pure, heralded singlephoton sources [J]. Optica, 2017, 4(1) : 90.

[14] BELHASSEN J, BABOUX F, YAO Q, et al. On-chip III-V monolithic integration of heralded single photon sources and beamsplitters $[\mathrm{J}]$. Applied Physics Letters, 2018, 112(7): 071105.

[15] WANG Jianwei, BONNEAU D, VILLA M, et al. Chip-tochip quantum photonic interconnect by path-polarization interconversion [J]. Optica, 2016, 3(4): 407.

[16] SIBSON P, ERVEN C, GODFREY M, et al. Chip-based quantum key distribution $[\mathrm{J}]$. Nature Communications, $2017,8: 13984$.

[17] WANG Hui, QIN Jian, DING Xing, et al. Boson sampling with 20 input photons and a 60 -mode interferometer in a 1014-dimensional hilbert space $[\mathrm{J}]$. Physical Review Letters, American Physical Society, 2019, 123 (25) : 250503.

[18] GUO Jianjun, LIANG Yao, HUANG Guang, et al. Pure dielectric waveguides enable compact, ultrabroadband wave plates $[\mathrm{J}]$. IEEE Photonics Journal, 2016, 8(5):
$1-9$.

[19] YAO Liang, ZHANG Fengchun, GU Jiahua, et al. Integratable quarter-wave plates enable one-way angular momentum conversion $[\mathrm{J}]$. Scientific Reports, 2016, 6: 24959.

[20] YANG Huizhan, ZHANG Jianhao, ZHU Yuntao, et al. Ultra-compact and temperature-insensitive Mach-Zehnder interferometer based on one multimode waveguide on silicon [J]. Optics Letters, 2017, 42(3): 615.

[21] NEGREVERGNE C, MAHESH T S, RYAN C A, et al. Benchmarking quantum control methods on a 12-qubit system [J]. Physical Review Letters, 2006, 96 (17): 170501.

[22] XIN Tao, HUANG Shilin, LU Sirui, et al. NMRCloudQ: a quantum cloud experience on a nuclear magnetic resonance quantum computer $[\mathrm{J}]$. Science Bulletin, 2018, $63(1)$ : 17-23.

[23] WANG Xilin, LUO Yihan, HUANG Heliang, et al. 18qubit entanglement with six photons' three degrees of freedom [J]. Physical Review Letters, 2018, 120(26): 260502.

[24] TANG Hao, LIN Xiaofeng, FENG Zhen, et al. Experimental two-dimensional quantum walk on a photonic chip [J]. Science Advances, 2018, 4(5) : 1-7.

[25] CIRAC J I, ZOLLER P. Quantum computations with cold trapped ions $[\mathrm{J}]$. Physical Review Letters, 1995, 74 (20) : 4091-4094.

[26] DIVINCENZO D P. Dogma and heresy in quantum computing $[\mathrm{J}]$. Quantum Information \& Computation, 2001, 1 (4) : $1-6$.

[27] WRIGHT K, BECK K M, DEBNATH S, et al. Benchmarking an 11-qubit quantum computer $[\mathrm{J}]$. Nature Communications, 2019, 10(1) : 1-6.

[28 ] WANG Ye, UM M, ZHANG Junhua, et al. Single-qubit quantum memory exceeding ten-minute coherence time [J]. Nature Photonics, 2017, 11(10) : 646-650.

[29] LU Yao, ZHANG Shuaining, ZHANG Kuan, et al. Global entangling gates on arbitrary ion qubits $[\mathrm{J}]$. Nature, 2019, 572(7769) : 363-367.

[30] D-wave System Inc. The D-wave 2000QTM system: the most advanced quantum computer in the world $[\mathrm{EB} / \mathrm{OL}]$. [2020-02-15]. https://www. dwavesys. com/d-wave-twosystem.

[31 ] PRESKILL J. Quantum computing in the NISQ era and beyond $[\mathrm{J}]$. Quantum, 2018, 2(1): 79.

[32 ] CHEN Zhaoyun, ZHOU Qi, XUE Cheng, et al. 64-qubit quantum circuit simulation $[\mathrm{J}]$. Science Bulletin, 2018, 63 (15) : 964-971. 
[33] SONG Chao, XU Kai, LI Hekang, et al. Generation of multicomponent atomic Schrödinger cat states of up to 20 qubits $[\mathrm{J}]$. Science, 2019, 365(6453) : 574-577.

[34] SHOR P W. Algorithms for quantum computation: discrete logarithms and factoring [C] // Proceedings 35th Annual Symposium on Foundations of Computer Science. New York, USA: IEEE Computer Society Press, 1996, 59 (3) : 124-134.

[35] GROVER L K. A fast quantum mechanical algorithm for database search $[\mathrm{C}] / /$ Proceedings of the 28th Annual ACM Symposium on Theory of Computing. New York, USA: ACM Press, 1996: 212-219.

[36] MONTANARO A. Quantum algorithms: an overview [J]. npj Quantum Information, 2016, 2(1) : 15023.

[37] MARTÍN-LÓPEZ E, LAING A, LAWSON T, et al. Experimental realization of Shor's quantum factoring algorithm using qubit recycling $[\mathrm{J}]$. Nature Photonics, $2012,6(11)$ : 773-776.

[38 ] VANDERSYPEN L M K, STEFFEN M, BREYTA G, et al. Experimental realization of Shor's quantum factoring algorithm using nuclear magnetic resonance $[\mathrm{J}]$. Nature, $2001,414(6866)$ : 883-887.

[39] KING J, YARKONI S, NEVISI M M, et al. Benchmarking a quantum annealing processor with the time-to-target metric $[\mathrm{EB} / \mathrm{OL}] .(2015-08-20)$ [2020-03-01]. https ://arxiv. org/abs/1508. 05087.

[40] CAI X D, WEEDBROOK C, SU Z E, et al. Experimental quantum computing to solve systems of linear equations [J]. Physical Review Letters, 2013, 110(23) : 230501.

[41] BARZ S, KASSAL I, RINGBAUER M, et al. A two-qubit photonic quantum processor and its application to solving systems of linear equations $[\mathrm{J}]$. Scientific Reports, 2014,4 : 6115.

[42] PAN Jian, CAO Yudong, YAO Xiwei, et al. Experimental realization of quantum algorithm for solving linear systems of equations $[\mathrm{J}]$. Physical Review A: Atomic, Molecular, and Optical Physics, 2014, 89(2) : 1-5

[43 ] LARSEN M V, GUO X, BREUM C R, et al. Deterministic generation of a two-dimensional cluster state $[\mathrm{J}]$. Science, 2019, 366(6463) : 369-372.

[44] HE Yu, DING Xing, SU Z E, et al. Time-bin-encoded boson sampling with a single-photon device $[\mathrm{J}]$. Physical Review Letters, 2017, 118(19) : 190501.

[45] ARUTE F, ARYA K, BABBUSH R, et al. Quantum supremacy using a programmable superconducting processor $[\mathrm{J}]$. Nature, 2019, 574(7779) : 505-510.

[46] YANG Mu, REN Changliang, MA Yuechi, et al. Experimental simultaneous learning of multiple nonclassical correlations $[\mathrm{J}]$. Physical Review Letters, 2019, 123 (19) : 190401.

[47] TOWNSEND P D, PHOENIX S J D, BLOW K J, et al. Design of quantum cryptography systems for passive optical networks [J]. Electronics Letters, 1994, 30(22) : 18751877

[48] BRASSARD G, BUSSIERES F, GODBOUT $\mathrm{N}$, et al. Multiuser quantum key distribution using wavelength division multiplexing $[\mathrm{C}] / /$ SPIE Proceedings: Applications of Photonic Technology 6. Quebec City, Canada: SPIE, 2003, 5260: 149-153.

[49] ELLIOTT C, COLVIN A, PEARSON D, et al. Current status of the DARPA quantum network $[\mathrm{C}] / /$ SPIE Proceedings: Quantum Information and Computation 3. Orlando, USA: SPIE, 2005 : 138-149

[50 ] PEEV M, PACHER C, ALLÉAUME R, et al. The SECOQC quantum key distribution network in Vienna $[\mathrm{J}]$. New Journal of Physics, 2009, 11(7) : 075001.

[51] CHEN Wei, HAN Zhengfu, ZHANG Tao, et al. Field experiment on a "star type" metropolitan quantum key distribution network $[\mathrm{J}]$. IEEE Photonics Technology Letters, 2009, 21(9) : 575-577.

[52] WANG Shuang, CHEN Wei, YIN Zhenqiang, et al. Field test of wavelength-saving quantum key distribution network [J]. Optics Letters, 2010, 35(14) : 2454-2456.

[53] CHEN Tengyun, WANG Jian, LIANG Hao, et al. Metropolitan all-pass and inter-city quantum communication network $[\mathrm{J}]$. Optics Express, 2010, 18 ( 26) : 27217-27225

[54] LI Yang, LIAO Shengkai, CHEN Xiele, et al. Spacebound optical source for satellite-ground decoy-state quantum key distribution $[\mathrm{J}]$. Optics Express, 2014, 22 (22) : 27281-27289.

[55] SASAKI M, FUJIWARA M, ISHIZUKA H, et al. Field test of quantum key distribution in the Tokyo QKD network [J]. Optics Express, 2011, 19(11) : 10387-10409.

[56] 王忠耀, 吴春燕. 粤港澳大湾区: 共担使命 同享荣光 [N]. 光明日报, 2019-02-20(10).

WANG Zhongyao, WU Chunyan. Guangdong-Hong KongMacao greater bay area: sharing the mission and sharing the glory $[\mathrm{N}]$. Guangming Daily, 2019-02-20(10). (in Chinese)

[57] XU Feihu, MA Xiongfeng, ZHANG Qiang, et al. Secure quantum key distribution with realistic devices $[\mathrm{J}]$. Reviews of Modern Physics, 2020, 92(2) : 025002.

【中文责编：英 子; 英文责编: 木 柯】 